\title{
Digitale Annotation alchemischer Decknamen
}

\author{
„Die Allegoriae werden uns nit mehr verborgen seyn.“
}

\begin{abstract}
This chapter aims to show the uses of modelling alchemical terms in a digital thesaurus using the example of Michael Maier's (1568-1622) writings. Alchemical language is supposed to be full of secrets and it is indeed full of ambiguities. They are revealed to initiated adepts ('experts') who are familiar with the underlying semiotic codes of analogy. Its allegories have wrongly brought alchemy the miscredit of being known as an 'esoteric pseudo-chemistry', which recent studies have proven wrong. Alchemical language is an example of scientia poetica; its Decknamen are coded, ornate and unstable. Computational methods like Natural Language Processing (NLP), Named Entity Recognition (NER) and knowledge representation technologies, for example using thesauri of terms of alchemy in XML, allow us to handle the typical ambiguity of alchemical data. We can make implicit instances of knowledge explicit in a digital thesaurus while the linking of a concrete word (a string or label) in a text to the thesaurus remains loose enough to allow for imprecise poetic language. Computational models are "temporary states in a process of coming to know", in which computers are not "knowledge jukeboxes" but "representation machines" (McCarty). They create a systematic approximation of reality, and from its shortcomings we learn about the reality we aimed to model. This might be a viable and helpful new approach in research on alchemy, a field which has shown a great reluctance to make meaning explicit in the past. But it also comes with the responsibility of ensuring that annotation remains as objective as possible, open to uncertainty and not too concrete in case of ambiguities. The main challenges are linking concepts and labels, and avoiding interpretation in the process of making information explicit, since the annotation will be done automatically.
\end{abstract}

Keywords: Automation, Disambiguation, Evaluation, Role of Interpretation, Classifying, Linking, Tagging, Ontologies, Digital Humanities, History of Science, Methodology

Sarah Lang, Projektassistentin des Geisteswissenschaftlichen Dekanats, Doktorandin am Zentrum für Informationsmodellierung, Universität Graz

2 Open Access. (c) 2020 Sarah Lang, published by De Gruyter. (c)) BY-NC-ND This work is licensed under the Creative Commons Attribution-NonCommercial-NoDerivatives 4.0 License.

https://doi.org/10.1515/9783110689112-010 


\section{Automatisierte Annotation und Alchemie}

Das Werk des Iatrochemikers Michael Maiers (1568-1622) umfasst ungefähr 3500 PDF-Seiten. ${ }^{1}$ Alchemischer Sprache haftet allerdings der Ruf an, voller Geheimnisse und ,verbal deceits' zu sein. ${ }^{2}$ Denn Leserinnen und Lesern ohne Vorerfahrung erschließen sich die sogenannten alchemischen Decknamen nicht ohne Weiteres; sie brauchen zusätzliche Information, um diese verstehen zu können. ${ }^{3}$ Bei Decknamen handelt es sich um eine Form indirekter Sprechweise, die typisch für alchemische Sprache ist, bei der chemische Substanzen durch bildhafte Metaphern umschrieben werden, wie etwa die Bezeichnung von Vitriol als ,der grüne Löwe'. Wie können nun diese 3500 Seiten auf eine effiziente Art und Weise dem Leser zugänglicher gemacht und ihre Decknamen, zumindest zum Teil, aufgelöst werden? Im Folgenden soll gezeigt werden, wie (halb-)automatisierte Annotation der Vorkommen solcher Signalwörter im Text in Verbindung mit einem Thesaurus, der zusätzliche Informationen zu den Konzepten enthält, unter Verfolgung eines regelbasierten Ansatzes dazu herangezogen werden kann. Der Beitrag reflektiert dabei die Herausforderungen und theoretischen Implikationen, die sich bei einer solchen (halb-)automatisierten Annotation ergeben.

Mit der digitalen Annotation wird es einerseits möglich den geisteswissenschaftlichen Aspekt der kontextualisierenden, erklärenden Hinzufügung maschinenlesbar zu machen. Andererseits bietet die automatisierte Annotation den Vorteil

\footnotetext{
1 Überblicksartig zur Person Michael Maiers: Leibenguth (2002); Tilton (2003). Bei der Zählung werden PDF-Seiten angeführt, da in Maiers Originaldrucken leider nicht alle Seiten nummeriert sind. Nachdem die Anzahl der nicht nummerierten Seiten aber recht groß ist, würde sich bei der ausschließlichen Zählung nummerierter Seiten eine deutlich zu geringe Anzahl ergeben. Hierbei ist natürlich zu beachten, dass dadurch auch leere Seiten, Scans von Einbänden, Titelblätter mit Informationen zum Datengeber des Faksimiles, etc. miteinberechnet wurden. Die genaue Anzahl an PDF-Seiten wurde, um dies auszugleichen, daher um ca. 5\% verringert. Es handelt sich bei der Angabe nur um eine Approximation, also um keine absolute Seitenanzahl.

2 Einführend zur Alchemie: Principe (2013). Die heutige Alchemiegeschichtsschreibung folgt zu großen Teilen der sog. ,New Historiography of Alchemy', die differenziertere Begriffsverwendungen und vor allem das Absehen von Anachronismen sowie überkommenen Forschungstopoi fordert. So handelt es sich bei der Bezeichnung der alchemischen Praktiker des 17. Jahrhunderts als ,Alchemisten' eigentlich um einen Anachronismus, da diese sich selbst als, Chymiker bezeichneten. Im Zuge dieser Begriffsrevidierung wurde auch dazu aufgerufen, gewisse Unterströmungen wie etwa die sog. transmutatorische Alchemie (Chrysopoeia) von der der Artzalchemisten (Iatrochemie) zu unterscheiden, wenn möglich und sinnvoll. ,Alchemie‘ wird aber dennoch weiterhin als Überund Sammelbegriff verwendet. Vgl. dazu Principe und Newman (2001).

$3 \mathrm{Zu}$ alchemischen Decknamen, vgl. Lippmann (1919); Ruska und Wiedemann (1924); Principe (1992); Newman (1996).
} 
einer großen Ersparnis an Zeit und Ressourcen, wenn die Zahl der Vorkommen so hoch wie bei Maier ist, wo man pro Seite mit 5-10 zu annotierenden Wörtern rechnen muss. Schließlich wird auch eine quantitative Textanalyse sinnvoll, die bei einer händischen Annotation zu viel Zeit und Ressourcen kosten würde.

Alchemische Texte bedienen sich einer Fachsprache, die für den modernen Leser nicht ohne Weiteres verständlich ist. Dazu muss für historische Alchemisten implizites Wissen für moderne Leser explizit gemacht werden. Zunächst stellt sich hierbei allerdings die Frage, welche Aspekte alchemischer Texte sich überhaupt dazu eignen, für eine zumindest halbautomatische Annotation formalisiert zu werden. Die historischen Autoren haben alchemische Decknamen auf innovative Art und Weise verwendet, und auch umfangreich neue Wörter geschaffen, doch gehören sie zumeist einem immer relativ ähnlichen finiten Fundus an. Einige solcher Allegorien verfestigten sich sogar zu stehenden Ausdrücken. Auch die Neologismen folgen den typischen Regeln alchemischer Bildhaftigkeit, indem die Beziehung zwischen Zeichen und Bezeichnetem in einer gemeinsamen, analogen Eigenschaft besteht. Ein repräsentativer Fundus solcher Decknamen lässt sich demnach in einem Thesaurus formalisieren. Die Annotation solcher Instanzen von implizitem Wissen ${ }^{4}$ soll als explizierende Hinzufügung dienen. Annotation mithilfe von Natural Language Processing und Wissensrepräsentation mithilfe eines Semantic Web Thesaurus als Knowledge Organization System können Licht in das „dunkle Vokabular“ (obscurum vocabulum) der Alchemie werfen, um implizites Wissen dieser Epoche heutigen Leserinnen und Lesern wieder besser zugänglich zu machen.

Die Annotation soll für den menschlichen Leser nützlich, aber auch für maschinelle Analyse effektiv verwendbar sein. Sie soll so gut als möglich automatisierbar, auf ein größeres Korpus alchemischer Literatur anwendbar sein und trotzdem geisteswissenschaftlichen Qualitätsansprüchen genügen. Doch beziehen sich Annotationen impliziten Wissens nicht auf einen für uns greifbaren Gegenstand, sondern einen historischen „Wissenshorizont“, den wir bestenfalls rekonstruieren können und wahrscheinlich im Prozess der Annotation auch zum Teil konstruieren müssen. Wie können Annotationen dabei möglichst, objektiv“ bleiben, aber dennoch aussagekräftig genug sein, um zur Disambiguierung uneindeutiger alchemischer Decknamen beizutragen? Eine weitere Herausforderung im Fall der Annotation impliziten Wissens stellt die Tatsache dar, dass das implizite Wissen in Konzepten (vgl. SKOS concepts) besteht, es im Text allerdings nur in Form von Wörtern bzw. Zeichenketten (vgl. SKOS labels) vorliegt (vgl. W3C-Consortium 2012). Wie und inwieweit kann Annotation diese Differenz sinnvoll oder überhaupt über-

4 Zum Begriff des tacit knowledge: Polanyi (2009). 
brücken? Diese Fragen sollen an konkreten Beispielen alchemischer Symbole und Decknamen bei Michael Maier diskutiert werden.

Denning schrieb 1985 ,The fundamental question underlying all of computer science is ,what can be automated?““(Denning 1985, 16) Beynon, Russ und McCarty stellen fest, dass es in Hinblick auf die Fortentwicklung von Technologie zweifellos sinnvoll sei, diese Frage zu stellen, aber meinen, sie betreffe und befördere geisteswissenschaftliche Erkenntnisinteressen nicht (vgl. Beynon et al. 2006, 150). Im Folgenden soll argumentiert werden, dass sehr wohl auch genuin geisteswissenschaftliche Analyseprozesse zumindest zum Teil automatisiert werden können, wenn man sich auf Aspekte beschränkt, die sich zu einer digitalen Formalisierung eignen. Zunächst wird eine Methode vorgestellt werden, die unter Hinzunahme eines formalen Wissensmodells über semi-automatische Annotation zu einer quantitativen Textanalyse bestimmter durch einen Thesaurus im Vorhinein spezifizierter Begriffe und ihrer Beziehungen untereinander führt. Dabei wird auf die Anforderungen eingegangen, die sich an die Erstellung eines solchen Thesaurus ergeben und die Herausforderungen, die darin liegen, Zeichenketten, die sich in Texten finden mit den Konzepten eines Thesaurus zu verbinden. Es wird die Frage angesprochen, warum sich gerade (halb-)automatisierte Annotation dafür eignet und nicht etwa bereits bestehende Methoden der quantitativen Textanalyse dazu ausreichen sowie die Frage, ob solche Annotation zum Erkennen literarischer Kontexte anwendbar ist. Weiterhin wird der genaue Ablauf der Annotation und eventuell dadurch möglich werdenden Disambiguierung erklärt sowie die Fragen nach Subjektivität und Evaluierungsmöglichkeiten solcher Annotationen angesprochen.

\section{Zur Methode: Automatisierte Annotation mithilfe eines Thesaurus}

Typisch für alchemische Texte ist ihre verrätselte Sprache, die sich besonders in der Verwendung von Symbolen und Decknamen äußert. Diese sollen im Zuge einer digitalen Edition über Verweise auf einen Symbolthesaurus zumindest teilweise erklärt oder verständlicher gemacht werden. Zudem sollen allerdings auch Beziehungen zwischen den Symbolen und Decknamen sichtbar gemacht werden. Die Wörter werden hierbei als ,Passwörter‘, als Ideenträger (vgl. Baudrillard 2000, 9-10) verstanden, in deren Hintergrund sich ein Wissenssystem konstituiert, das in einem Thesaurus abgebildet werden kann. Alchemische Fachsprache, in ihrer uneigentlichen Rede, beinhaltet ,implizites Wissen' (tacit knowledge). Doch dieses implizite Wissen kann zum großen Teil durch Methoden der digitalen Wissensre- 
präsentation wieder explizit gemacht werden (vgl. Collins 2010). Linked Data unter Nutzung von Semantic Web Technologien stellen eine dafür besonders geeignete Form dar (vgl. Oldman et al. 2016, 258, 266-267).

Eine wichtige Aufgabe des Vorhabens ist, aus den Indices und Registern der Werke Michael Maiers einen Thesaurus zu erstellen, der Maiers ,Wissenshorizont“ möglichst verlässlich wiedergibt. Der zu erstellende Thesaurus dient als Knowledge Organization System (KOS), das mit dem Semantic Web-Vokabular SKOS (Simple Knowledge Organization System, W3C-Consortium [2012]) kodiert werden soll. Das W3C hat SKOS als Standard vorgeschlagen, und das Vokabular ist in leicht abgewandelter Form auch als ISO 25964 normiert (vgl. Stock und Stock 2008, 228-255). Auch der Alchemie-Thesaurus, der an der Herzog August Bibliothek Wolfenbüttel bereits erstellt wurde (Frietsch 2017a, 2020), soll in dieses weiterverwertbare Datenformat überführt und inhaltlich ergänzt werden. ${ }^{5}$ Anhand der genannten Werke Michael Maiers soll dann einerseits automatisiertes Tagging mithilfe dieses Thesaurus erprobt werden und andererseits der Thesaurus anhand der Vorkommnisse potentieller Decknamen in Maiers Werk vervollständigt werden.

Unter dem Kampfbegriff der ,New Historiography of Alchemy“ ist eine neue Herangehensweise an Alchemiegeschichte entstanden. Geleitet durch eine Zurückweisung anachronistischer Begrifflichkeiten, die sich in der Alchemieforschung etabliert hatten, hat diese sich zum Ziel gesetzt, einerseits durch eine präzisere Begriffsverwendung im Sinne der historischen Quellen und andererseits durch eine tiefergehende Erforschung alchemischer und chymischer Begrifflichkeiten und Kommunikationsweisen wie z. B. der sogenannten Decknamen Alchemiegeschichte zu schreiben, ohne auf anachronistische Kategorien wie z. B. die Bezeichnung der Alchemie als Pseudowissenschaft zurückzugreifen und gleichzeitig die Alchemie als historische Wissenschaftsform besser zu verstehen. ${ }^{6}$ Bei der Analyse solcher Decknamen im Sinne von L. Principe und W. Newman werden ,dunkle‘ Begriffe chemisch gelesen und im Zuge einer Nachstellung im Sinne der Experimentalarchäologie einem Kohärenzbeweis unterzogen (vgl. Principe 1992; Newman 1996). Ergibt der ,dunkle‘ Begriff nach seiner Übersetzung in eine chemische Versuchsanordnung sowohl theoretisch auch praktisch Sinn, so wird der Deckname im Sinne eines ,best explanation'-Ansatzes nach dem Kohärenzbeweis als ent-

5 Ein Thesaurus dient der Wissensorganisation und -repräsentation. Kodiert im Semantic Web wird er maschinenlesbar, die abgebildeten Konzepte durch URIs eindeutig referenzierbar; weiters erlaubt SKOS deren Dokumentation und Definition durch Zusatzinformationen sowie die Darstellung von Verbindungen, Hierarchien und Synonymien, die eindeutige Klassifikation erlaubt, aber dennoch nicht zu rigide ist. Vgl. Yu (2015, 156-263) .

6 Zur (mitunter kontroversen) Diskussion um die „New Historiography of Alchemy“: Newman und Principe (1998); Principe und Newman (2001); Tilton (2003, 9-18) 
schlüsselt angesehen. Dieser Zugang erlaubt der Forschung, sowohl ein vertieftes Verständnis über alchemische Decknamen und Sprechweisen zu erlangen, als auch sich in naturwissenschaftlicher Art und Weise über den Wissensstand historischer AkteurInnen klar zu werden und schließlich experimentell nachzuvollziehen, wie ein Deckname und das realweltliche Ding, das er beschreibt, zueinander in Beziehung stehen. Ein solcher Beweis sollte jedoch auch über Logik möglich sein, da es sich dabei um einen Kohärenzbeweis handelt. Essentiell ist hierbei die Frage, wie Zeichen und Bezeichnetes in Relation zueinander gebracht werden können.

Zur alchemischer Sprache haben z. B. Hans-Werner Schütt und Umberto Eco schon einige Überlegungen beigetragen, doch sind diese meist theoretischer Natur und gehen über Forschungstopoi kaum hinaus, weswegen sie für konkrete Textanalysen nicht fruchtbar zu machen sind. ${ }^{7}$ Bereits bestehende Ressourcen zu alchemischen Begrifflichkeiten waren zur Information über Alchemie, aber nicht zur Annotation konkreter alchemischer Texte gedacht, wie z. B. das Alchemie-Lexikon (Priesner und Figala 1998) oder der Alchemie-Thesaurus der HAB Wolfenbüttel, der der Verschlagwortung und Erschließung alchemischer Buchbestände diente. ${ }^{8}$ Die bisher vorhandenen Ressourcen erlauben zwar ein Einarbeiten in oder Vorinformieren über Alchemie und gewisse alchemische Konzepte durch Einlesen, sind aber nicht für eine automatisierte Annotation konkreter alchemischer Texte geeignet. Die dort vorkommenden Einträge sind nämlich häufig allgemeine Überbegriffe, die so in den Texten gar nicht behandelt werden, doch finden sich unter den Lemmata keine Zeichenketten, die in den Texten selbst verwendet werden. ${ }^{9}$ Es bedarf demnach sowohl einer neuen Art des Sprechens über alchemische Sprache als auch eines neuen Thesaurus, die beide mit dem Ziel der automatisierten Annotation im Hinterkopf konzipiert wurden. Der hier vorzustellende Ansatz einer automatisierten Annotation alchemischer Texte mithilfe eines Thesaurus verwendet aus pragmatischen Gründen einen sehr weiten Decknamenbegriff: Als Deckname wird in diesem Kontext jede Zeichenkette verstanden, die mit einiger

7 Z. B. Schütt (1994); Eco (2016), zu chemischer Sprache im 18. Jahrhundert dann auch Duncan (1981).

8 Ein wenig problematisch an der Benennung dieses Thesaurus ist der Umstand, dass es sich hier um eine nicht dem allgemeinen Verständnis von „Thesaurus“ folgende Bezeichnung handelt. Frietsch (2017a); Frietsch (2017b); Frietsch (2020).

9 So ergab beispielsweise eine automatisierte Annotation der Symbola Maiers, dass von den 99 Einträgen des HAB-Thesaurus gerade einmal 19 überhaupt in Maiers Werk angefunden wurden, da die meisten Begriffe nur zum Sprechen über Texte geeignet sind und abstrakte Forschungsthemen bezeichnen, die natürlich im Klartext der Quellen nicht so bezeichnet stehen. Es werden daher einerseits viele Begriffe des HAB-Thesaurus in der Quelle nicht angefunden, andererseits bleiben viele erklärungsbedürftige Konzepte des Maier-Textes unerklärt, da sie wohl zu speziell gewesen wären, um sie im besagten Thesaurus abzubilden. 
Wahrscheinlichkeit ein Deckname sein könnte. ${ }^{10}$ Ob diese Zeichenkette vom Autor zur tatsächlichen Kodierung alchemischen Wissens gedacht war, kann die automatisierte digitale Methode nicht beurteilen. Die Entscheidung und Interpretation obliegt weiterhin den FachwissenschaftlerInnen. Doch die vorgestellte Methode erlaubt, die Wörter, die Kandidaten für Decknamen sind, zu kontextualisieren und Wahrscheinlichkeiten zur Disambiguierung ambivalenter Begriffe anzubieten. Die Zeichenketten der ,Decknamen-Wörter‘ werden hier nicht als Wörter verstanden, die mithilfe eines Wörterbuchs einfach ,aufgelöst' werden können, sondern als bloße Wortoberflächen (Zeichen), die auf Begriffe (Bezeichnetes) verweisen, auf die aus dem Text heraus referiert wird. Diese Auffassung erlaubt das Verständnis alchemischer Sprache als Fachsprache, da die Decknamen nicht als Terme, sondern lediglich als Hinweiswörter verstanden werden. Wenn der Term in der modernen Terminologie eindeutig auf einen durch ihn bezeichneten Begriff verweist, so erlaubt ein Deckname Verweise in analogischen Assoziationsketten, über die Verbindungen zwischen Zeichen und Bezeichnetem hergestellt und beschrieben werden können.

Thesaurus-Begriffe werden aus den Indices der Werke Maiers ausgewählt und durch solche ergänzt, die zur Schaffung von Assoziationsketten benötigt werden. So führt Maier z. B. keine Farben an, die jedoch als Qualifier in dem Modell unerlässlich sind, weil sie häufig die Eigenschaften bezeichnen, über die die Assoziation zwischen Zeichen und Bezeichnetem hergestellt wird. Daraufhin werden die fraglichen Zeichenketten im Korpus automatisiert annotiert. Daraus wiederum wird eine Konkordanz der Wortverwendung erstellt. Diese Konkordanz wird abermals mit denselben Zeichenketten annotiert, wodurch sich ein Beziehungsnetzwerk ergibt, das Aussagen darüber zulässt, in welchen inhaltlichen Kontexten ein Begriff auftritt. Diese ergeben sich selbst aus sprachlichen Kontexten der konkreten Textstelle. Begriffe im Thesaurus können somit mit Kontexten getaggt werden. Diese Kontexte allerdings sind quantifizierbar, d. h. durch einen Vergleich der Mittelwerte für die Kontexte in der Konkordanz mit den Prozentsätzen an einer konkreten Textstelle kann eine Wahrscheinlichkeit zur Disambiguierung der Wortverwendung

\footnotetext{
10 Principe definiert Decknamen wie folgt: „To promote [...] secrecy, Zosimos employs a technique that would become typical for alchemical authors: the use of Decknamen, a German term meaning ,cover names'. These Decknamen function as a kind of code. Instead of using the common name for the substance, the alchemical writer substitutes another word - usually one that has some link, literal or metaphorical, with the substance intended. [...] Decknamen serve a dual purpose: they maintain secrecy, but they also allow for discreet communication among those having the knowledge or intelligence to decipher the system. They simultaneously conceal and reveal. Consequently, Decknamen have to be logical, not arbitrary, so that they can be deciphered.“ Principe $(2013,18)$.
} 
angeboten werden. Weiterhin hat diese Methode den Vorteil, dass sie ein relativ großes Maß an Objektivität mit sich bringt. Einen subjektiven Eingriff stellt dabei lediglich die Entscheidung dar, welche Begriffe in den Thesaurus aufgenommen werden. Die Definition der Begriffe selbst wird größtenteils durch die Konkordanz geliefert, wodurch quasi ein Buch das andere erklärt, beziehungsweise die Gesamtmenge der Schriften des Autors selbst eine konkrete Textstelle kontextualisiert. Diese Methode könnte auch Hinweise darüber liefern, ob ein Autor die Technik der Wissensdispersion verwendet, da Vorkommen gewisser Signalwörter sehr schnell überblickbar werden. ${ }^{11}$ Thesaurus, Konkordanz, Korpus und konkrete Textstelle werden miteinander in Verbindung gebracht. Der Thesaurus bringt somit als eine Art Gesamtregister Wissen, das im Zuge von Wissensdispersion absichtlich über mehrere Texte verteilt wurde, wieder zusammen. Die Verbindungen beschreiben ein Wissensnetzwerk, das somit digital modelliert wird.

Ein solches Modell ist weder in Stein gemeißelt noch will es ,Wahrheiten' vermitteln. Digitale Modelle definiert McCarty als ,temporary states in a process of coming to know“, in dem Computer nicht „knowledge jukeboxes“, sondern „representation machines“ sind (McCarty 2004, 255). Aus einem Modell lernen wir genau an den Stellen, wo das Modell nicht auf die Realität passt. Dabei wird ein zirkulärer Prozess der Modellierung angestoßen, im Zuge dessen mit jedem Zyklus das Verständnis über die zu modellierenden Aspekte, und damit auch das Modell selbst, verfeinert wird. Das Modell hilft also nicht nur dabei, konkrete Stellen im Text Michael Maiers besser verständlich zu machen, sondern vermittelt auch Einsichten in das Funktionieren alchemischer Sprache. Der Thesaurus ist erweiterbar und kann somit durch die Ergebnisse zukünftiger digitaler Projekte ergänzt werden. Wenn diese allerdings auch funktionieren wie z. B. die zuvor angesprochenen, so könnten wieder keine direkten Verbindungen zwischen den digitalen Lexikon-Ressourcen und den transkribierten Texten gezogen werden. Doch neben diesen Projekten gibt es zudem eine Vielzahl historischer Alchemie-Wörterbücher, besonders aus dem 17. und frühen 20. Jahrhundert, sowie einige im Zuge des ,new historiographical turns‘ in der Alchemiegeschichte seit den 1990ern entstandene. Auf Dauer wird es ohnehin unerlässlich werden, diese als digitale Ressourcen ver-

11 Dabei handelt es sich um die Technik, essentielle Informationen an einer Stelle zur Wahrung der Geheimhaltung auszusparen. Von alchemisch vorgebildeten Lesern wird allerdings erwartet, dass diese einerseits das Fehlen einer essentiellen Information aufgrund ihrer chemischen Erfahrung bemerken und andererseits, dass sie die sonstigen Schriften des Autors auf der Suche nach dieser Information zu durchforsten wissen. Diese findet sich zumeist in einem ganz anderen Kontext ,versteckt' und kann häufig daran erkannt werden, dass gewisse beiden Stellen gemeinsame Signalwörter fallen. So scheint es wahrscheinlich, dass die Werke Maiers, die unterschiedlichste Themen behandeln, solche Wissensdispersion enthalten könnten. 
fügbar zu machen und als Linked Open Data zu synthetisieren. Die hier vorgestellte Methode liefert dabei das Bindeglied, mit dem eher abstrakte Begriffsdefinitionen mit tatsächlich in Texten vorkommenden Strings verbunden werden können. Auch historische Alchemie-Lexika, wie z. B. das Lexicon Alchemiae Rulandi könnten in das digitale Abbild des Wissensnetzwerks integriert werden (vgl. Ruland 1612). Der Zirkularität und Erweiterbarkeit der Modellierung wird dadurch Rechnung getragen, dass der mit der vorgestellten Methode zu verarbeitende Text nicht mit ,vergänglichen' Annotationen angereichert wird. Er enthält lediglich Bindeglieder zu einem Standoff-Thesaurus, ${ }^{12}$ über den Assoziationsketten und Bindeglieder je nach Status der Modellierung dynamisch ausgelesen werden können.

\section{Zum automatisierten Annotationsvorgang}

Die Datenakquisition erfolgt über die Software Transkribus. Mit einem im Zuge des NOSCEMUS-Projektes trainierten Modell liefert diese OCR-Transkriptionen sehr guter Qualität mit einer Fehlerquote von circa 1-3 Fehlern pro Seite. ${ }^{13}$ Dabei wird ein Korpus der gesamten Druckwerke Michael Maiers erstellt, das in der Folge zur Kontextualisierung von Textstellen herangezogen werden kann. Die Auswahl der $\mathrm{zu}$ annotierenden Inhalte orientiert sich an den Indices, die einigen Maier-Werken beigegeben sind: Aus den Index-Einträgen wird eine Liste kompiliert. ${ }^{14}$ Duplikate werden entfernt. Eine Sortierung in Named Entities und Konzepte wird händisch

12 Mit Standoff-Annotation verbinden Gius und Jacke die Vorstellung, dass die Annotation weniger normative und zeitlose Gültigkeit beansprucht als dies beispielsweise in einer Inline-Annotation der Fall wäre. Vgl. Gius and Jacke (2015). Im Text werden nur gewisse Strings annotiert. Erst über die Verbindung mit dem Thesaurus wird Zusatzinformation hinzugefügt. Welche Zusatzinformation hinzugefügt wird ist damit im eigentlichen Text nicht enthalten, wodurch diese laufend aktualisiert und verbessert werden kann, ohne dass am eigentlichen annotierten Text etwas verändert werden müsste. Der Begriff Standoff wird hier nicht im Gegensatz zu „embedded“ verstanden, sondern verweist nachdrücklich auf den Umstand, dass der Thesaurus als nicht direkt Teil als Annotation an den Text gedacht wird: Die eigentliche Annotation besteht in der Markierung der verweisenden Zeichenketten im Text, die in der Folge genausogut durch einen anderen Thesaurus erklärt werden könnten. Der Begriff Standoff dient dazu, die den nicht zwingenden Charakter der Verbindung zwischen annotiertem Text und Thesaurus herauszustellen.

13 Zum NOSCEMUS-Modell der News-Eintrag ,15/12/2019: Noscemus Transkribus Model released‘: https://www.uibk.ac.at/projects/noscemus/ (06.01.2020).

14 Zum Beispiel enthalten Maiers Symbola bereits über 3000 Index-Einträge, von denen allerdings einige Mehrfachnennungen aussortiert werden müssen. Die Streuung der relativen Häufigkeiten dieser Begriffe ist natürlich relativ groß. Vgl. Maier (1617, PDF-Seiten 661-702, 40 unnummerierte Seiten). 
vorgenommen. Von diesen werden auch einige wie Konzepte in den Thesaurus aufgenommen, andere wiederum dienen hauptsächlich bibliographischen Nachweisen. Sie können zum Nachvollziehen der Quellen Maiers herangezogen werden, doch hat der Umstand, welche antiken Historiographen Maier zitiert, vergleichsweise wenig Bewandtnis für die Interpretation seiner chymischen Theorien.

Zitate im Text werden händisch als solche ausgezeichnet, um die Wortverwendung in Zitaten von der im Haupttext unterscheiden zu können. So kann festgestellt werden, ob dies unterschiedliche Bewertungen der Decknamen ergibt. Im Thesaurus wird aus den Indices zunächst eine Liste an zu annotierenden labels erstellt. Schritt für Schritt werden zu diesen Konzepte hinzugefügt, wobei allerdings selektiv vorgegangen wird. Eine Zeichenkette kann als label verzeichnet mit Kontexten getaggt werden, muss aber nicht zwangsläufig durch die Hinzufügung eines verbundenen Konzepts noch tiefer erschlossen werden. Diese Erschließung findet nur in Bezug auf besonders relevante Konzepte statt, bei denen auch genug Aussagen Maiers vorhanden sind, um überhaupt eine solche Erschließung vorzunehmen. Ein besonderer Fokus liegt dabei auf den sieben Metallen, die Maier im Viatorium sehr ausführlich beschreibt (Maier 1618). Da die Indices sich besonders auf Personennamen konzentrieren, werden chemische Fachbegriffe und andere zur Kontextualisierung notwendige Elemente wie etwa Farben hinzugenommen. Die labels des Thesaurus werden im Text lemmatisiert gesucht ${ }^{15}$ und alle gefundenen Vorkommen automatisch im Stil von <fund label="normalisierter_Labelname" $>$ annotiert. Aus dem annotierten Korpus wird nun für alle labels eine Konkordanz aus den Annotationen erstellt. Der Text der Konkordanz wird wiederum mit den Begriffen des Thesaurus annotiert, wodurch ein Begriffsnetzwerk entsteht. Im Thesaurus werden Begriffen Kontexte zugewiesen.

\section{Warum Annotation und nicht quantitative Textanalyse?}

Es stellt sich eine weitere Frage: Wenn ohnehin automatisiert vorgegangen werden soll, warum dann nicht gleich auf bereits existente Methoden der quantitativen Textanalyse zurückgreifen? Hier soll argumentiert werden, dass Methoden der quantitativen Textanalyse, die nicht spezifisch auf die Analyse alchemischer Texte hin optimiert sind, nur relativ schwache Ergebnisse erzielen und damit nur sehr

15 Natural Language Processing Arbeitsschritte wie die Lemmatisierung werden mithilfe des Python Classical Language Toolkit (CLTK) umgesetzt. Cf. Johnson et al. (2014-2019). 
spezielle Forschungsfragen beantwortbar werden, die wissenschaftshistorisch nicht unbedingt besonders relevant oder interessant sind. Wenn automatisierte Annotation auf ein ausreichend großes Korpus angewendet wird, können die Resultate dann noch immer quantitativ ausgewertet und beispielsweise abschließend zu einer zusätzlichen Visualisierung verarbeitet werden. ${ }^{16}$

Standardanwendungen der quantitativen Textanalyse lassen keine gezielte Analyse wissenschaftlich relevanter Termini in ihren Relationen zueinander zu. ${ }^{17}$ So vermittelt linguistisches Keyword in Context ${ }^{18}$ keinen Zugang zum wissenschaftlichen Begriff im Kontext anderer wissenschaftlicher Konzepte. Topic Modelling ${ }^{19}$ scheint auf den ersten Blick eine inhaltliche Kontextualisierung zu erlauben, doch stellt sich hier das Problem, dass zur Erstellung eines ,allgemeingültigen' Kontexts für ein Wort oder Topic zunächst im Bag of words die tatsächlichen textuellen Kontexte verworfen werden. Zur Analyse eines extrem ambivalenten alchemischen Begriffs wie beispielsweise im Fall des bedeutungsreichen ,Mercurius “ wird der String bei jedem Vorkommen als derselbe behandelt, obwohl in den konkreten Situationen gar nicht vom selben Konzept die Rede sein mag. Diese „proximity to the source text“ ist es auch, die McCarty als einen ganz entscheidenden Vorteil der Annotation ansieht (vgl. McCarty 2003, 3). Die Disambiguierung, wie sie ja auch in modernen Sprachen und alltäglichen Kontexten, so beispielsweise im Fall des String ,Bank' notwendig ist, stellt sich hier komplexer heraus, da wir bisher keine statistischen Daten darüber haben, die relativ sicher Auskunft darüber vermitteln können, in welchem Kontext welche Bedeutung auftritt. Im Fall der alchemischen Termini kennen wir zum Teil nicht einmal das komplette Spektrum möglicher Bedeutungen und werden womöglich dazu auch gar nie in der Lage sein, da das Schaffen von Neologismen oder bewusst kreative und übertragene Wortverwendungen ein Spezifikum alchemischer Redeweise ausmachen. Ein regelbasierter Ansatz sollte daher hier einem statistikbasierten überlegen sein, zumal uns zumindest die Logik der alchemischen Sprechweise bekannt ist, wir aber zur massenhaften Analyse wohl ein sparse data-Problem zu erwarten haben. Auch für

16 So wurde im To See Or Not To See-Webtool ebenfalls ein quantitatives Visualisierungstool für Charakteristika von Shakespeare-Stücken entwickelt, dessen Netzwerkanalysen auf Annotationen beruhen. Vgl. Wilhelm, Burghardt und Wolff (2013). Ähnliche Visualisierungen könnten auch zur interaktiven Explorierung alchemische Begriffe nützlich sein.

17 Die Notwendigkeit, spezifisch für konkrete historische Fragestellungen nützliche Tools zu entwickeln, statt Out-of-the-box-Methoden zu nutzen betonen auch Gibson et al. (2019, 500-501). 18 Zur Keyword in Context (KWIC) Methode: https://fortext.net/ressourcen/glossar (06.01.2020). 19 Einführend zum Topic Modelling: Jan Horstmann (2018, § 2): „Topic Modeling“. In: forTEXT. Literatur digital erforschen. URL: https://fortext.net/routinen/methoden/topic-modeling (06.01.2020). 
andere Problematiken, die sich bei einer solchen Analyse ergeben können, wie z. B. den Umgang mit Kapitelgrenzen oder Vorkommnisse von Begriffen innerhalb von Zitaten anderer Autoren, ist Annotation deutlich besser geeignet als quantitative Textanalyse. Außerdem bleibt durch die Annotation besser nachvollziehbar, wie der Computer zu einer Interpretation gekommen ist, als bei einer statistischen Datentransformation aus einem Bag-of-words-Modell.

\section{Noscitur e sociis: Können literarische Kontexte automatisiert erkannt werden?}

Wenn wir die Frage stellen, ob literarische Kontexte automatisiert erkannt werden können, so kann dies beispielsweise leicht mit ,ja‘ beantwortet werden, wenn wir nur unsere Zieldefinition entsprechend anpassen. Linguistisch gesehen bietet Keyword-In-Context (KWIC) genau dies. ${ }^{20}$ Doch kann diese Definition auch auf literaturwissenschaftliche oder historische Fragestellungen übertragen werden? Die Antwort hängt davon ab, was wir als relevanten Kontext definieren.

Im Gegensatz zum ,Bank'-Disambiguierungsproblem, wobei die unterschiedlichen Bedeutungen doch in den meisten Fällen deutliche Änderungen im direkten Umfeld des linguistischen KWIC nach sich ziehen, so ist dies im Fall des alchemischen ,Mercurius‘ nicht unbedingt der Fall: Es kann passieren, dass die konkrete Chemikalie Quecksilber oder aber das philosophische Konzept Mercurius dieselben Eigenschaften besitzen und mit dem selben Kontext-Vokabular angesprochen sind und umgeben werden. In diesem Fall bildet sich der Bedeutungsunterschied maximal in einem sehr weiten KWIC ab, das dann allerdings eine automatisierte

\footnotetext{
20 McCarty äußert sich zurückhaltend zum Nutzen des KWIC-Prinzip des noscitur e sociis für die Literaturwissenschaft: , In J. R. Firth’s happy formulation, the underlying principle is that ,You shall know a word by the company it keeps!‘ $(1957,11)$. Marshalling and enumerating this company, concordance software has already helped us to inch forward by resolving context partially into particular words, their patterns of collation and a theoretical entity called ,span', within which collocation is probable. [...] Literary scholars are less well served by concordance software than linguists because literature adds to the linguists' rather amorphous idea of ,running text' a complex host of structural relations within and among individual texts. The analytic purposes of the literary scholar go beyond the ancient legal principle of noscitur e sociis which Firth is invoking, and so well beyond the capabilities of concordance software as we now know it." McCarty (2007). Die vorgestellte Methode versucht, eben dieser Sorge McCartys vorzubeugen, dass ein rein linguistisches KWIC nicht den Ansprüchen der Literaturwissenschaft genügt. Durch das Etablieren eines „Decknamen“-Kontexts werden relevante Elemente aus einem weiteren Kontext mit herangezogen.
} 
Klassifizierung erschwert, da die BearbeiterInnen womöglich die Textstellen eigens durchlesen müssten, um zu einem Urteil zu kommen. Ich schlage vor, einen ,Decknamen-Kontext` zu bilden. Darin dienen beieinanderliegende Annotationen als Kontext-Wörter, nicht einfach nur irgendwelche direkt umliegenden Wörter. Zum Beispiel könnten die fünf vorhergehenden und die fünf direkt nachfolgenden Annotationen als solche Kontexte angesehen werden. Der ,Decknamen-Kontext ${ }^{*}$ kann in deutlich mehr Fällen Aufschluss über die Bedeutungsfärbung alchemischer Texte vermitteln. Eine Konkordanzansicht kann wie in Listing 1 aussehen.

Listing 1: Textbeispiel von S. 217 der Arcana Arcanissima Maiers zu Odysseus

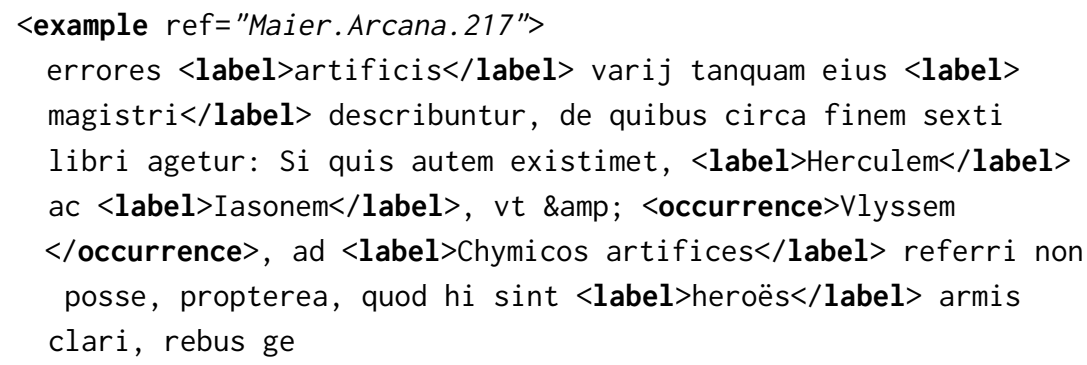

In Beispiel ,Maier.Arcana.217‘ wird der Begriff ,Ulysses“ kontextualisiert (<occurrence>). Als label sind alle im Kontext vorkommenden anderen ThesaurusBegriffe annotiert. Diese beinhalten z. B. , artifici is‘, ,magistri“ oder ,Chymicos artifices', Bezeichnungen, die stark darauf hinweisen, dass hier aus einer Meta-Perspektive über das alchemische Werk diskutiert wird. Zusätzlich ist hier ein mythologischer Kontext vertreten: Mit ,Herculem', ,Iasonem und ,heroes“ wird ein Kontext mythologischer Helden etabliert. Die Kombination der beiden vorhandenen Kontexte kann dahingehend interpretiert werden, dass Maier hier mythologische Helden als Allegorien für alchemische Adepten heranzieht. Tatsächlich ergibt ein close reading, dass dem so ist (Forshaw 2019; Lang 2018, 95-105). Auch andere Textstellen zu Odysseus ergeben ähnliche Kontexte. Die Zeichenkette ,Odysseus‘ kann demnach wie in Listing 2 im Thesaurus vermerkt werden.

Listing 2: Pseudo-Code, der die gefundenen Eigenschaften aus Listing 2 in RDF-Tripeln ausdrückt :Odysseus : hasContext :mythological, :alchemicalopusMeta .

Im vorliegenden Beispiel ist das konkrete Vorkommen ,Ulyssem‘ zu 50\% mit Kontext ,alchemicalOpusMeta' und zu 50\% mit ,my thological' getaggt. Daraus könnte 
auch ohne close reading mit relativer Sicherheit gefolgert werden, dass es sich bei diesem Decknamen nicht um eine konkrete chemische Substanz handelt. Welche Bedeutung hier genau intendiert wird, bleibt den interpretierenden HistorikerInnen zu entscheiden überlassen. Eine Disambiguierung bezüglich für die Analyse alchemischer Texte relevanter Aspekte konnte dennoch bereits erzielt werden.

In einem anderen Beispiel soll ein kurzer Blick auf,Mercurius' geworfen werden, der wohl den schwierigsten und vieldeutigsten Begriff der Alchemie darstellt:

Listing 3: Textbeispiel von S. 60 der Arcana Arcanissima Maiers zu Mercurius

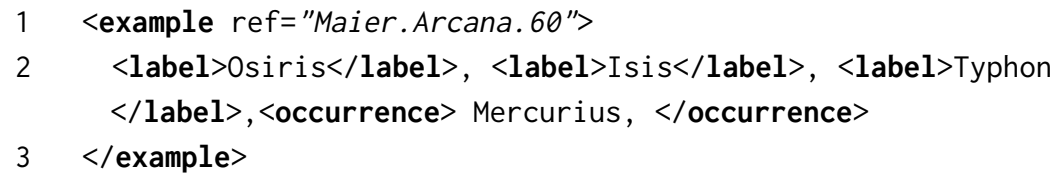

Aus ,Maier. Arcana.60' werden ,Osiris‘, ,Isis‘ und ,Typhon“ jeweils sowohl mit ,mythological' als auch mit dem Kontext ,alchemical innovators‘ getaggt, da diese in der Alchemiegeschichte mitunter als wichtige Akteure der eigenen Tradition verstanden wurden. Als solche werden sie eher als historische Personen denn als Angehörige dessen verstanden, was für uns heutzutage Mythologie bedeutet. In diesem Fall kann also geschlossen werden, dass die Klassifizierung des Kontexts ,my thological' überwiegt, da alle vorkommenden Begriffe auch als ,alchemical innovators' getaggt sind. Es geht also an dieser Stelle mit größerer Wahrscheinlichkeit um ,Mercurius‘ in seiner Rolle als ,Hermes Trismegistos‘, einen oder sogar den ,Urvater' der Alchemie.

Listing 4: Textbeispiele von S. 191 und S. 192 der Arcana Arcanissima Maiers zu Mercurius

2

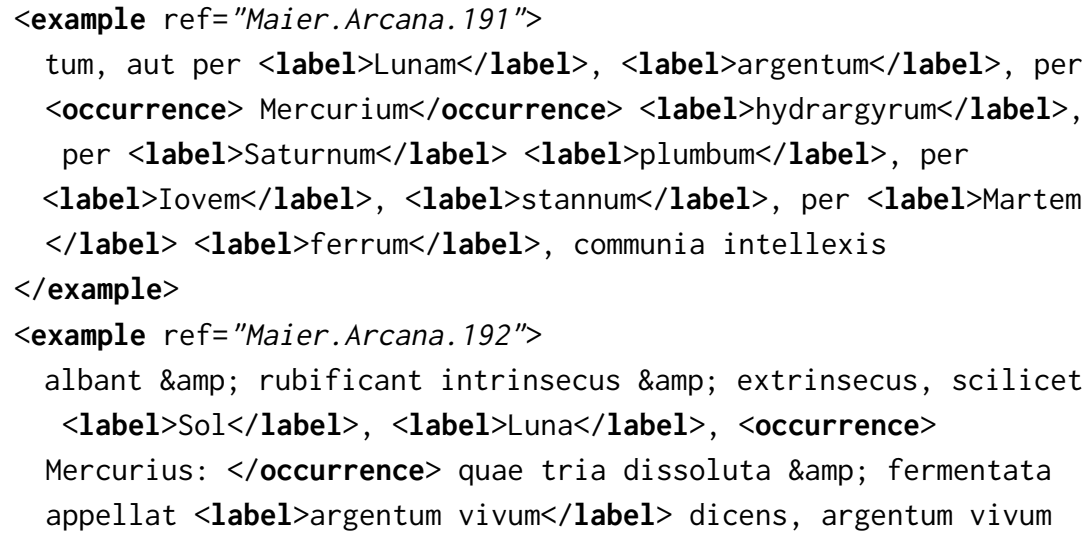


habet in se $<$ label $>$ corpus $</$ label $>$, <label $>$ spiritum</label $>$

\&amp; <label>animam</label $>$.

In ,Maier . Arcana. 191' und ,Maier . Arcana. 192“ handelt es sich um einen ,metallischen' Kontext: ${ }^{21}$ Nachdem die Textstellen nahe beieinander liegen, können wir davon ausgehen, dass wir uns an dieser Stelle in einem ,gemeinsamen' Kontext bewegen. Das Beispiel von Seite 191 enthält mythologische Namen, die auch als Planetenbezeichnungen verwendet werden (z. B. ,Saturnum' sowie Metallbezeichnungen in unverhüllter Rede wie ,argentum‘, ,hydrargyrum‘, etc.):

Listing 5: Pseudo-Code, der die gefundenen Eigenschaften aus Listing 4 auf der vorherigen Seite in RDF-Tripeln ausdrückt

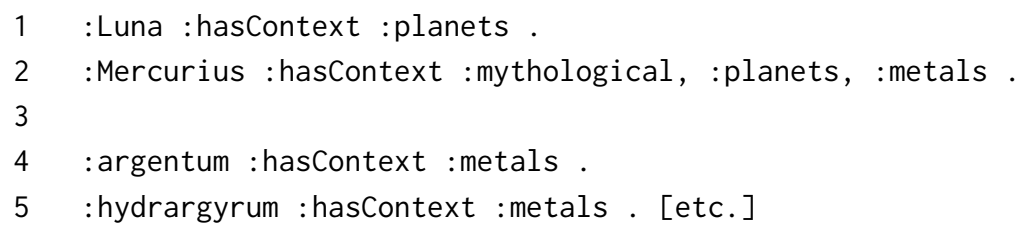

Eine Auszählung ergibt somit 5x :planets, 9x :metals und 4x :mythological. Es kann also eindeutig festgestellt werden, dass es hier um Metalle geht, die im Kontext von Planetennamen diskutiert werden. Das Lesen der Textstelle bestätigt eben diese Feststellung. Wie an diesem Beispiel ersichtlich wird, ergibt die Auswertung in vielen Fällen noch keine neuen Erkenntnisse. Die Mächtigkeit der Methode kommt erst zum Tragen, wenn es darum geht, einen Begriff über ein sehr großes Korpus zu verfolgen und beispielsweise festzustellen, ob er vorwiegend für konkrete Chemie oder eher philosophische Konzepte verwendet wird. Das Beispiel ,Odysseus` ergibt hier sehr eindeutige Ergebnisse, da er im Grunde nie in wirklich chemischen Kontexten auftritt. ${ }^{22}$ Dies könnte die vorgestellte Methode in kürzester Zeit feststellen. Durch die Nähe von Annotationen und Konkordanz zum Originaltext wird auch die händische Nachkontrolle durch fachkompetente ForscherInnen im Sinne eines close reading beträchtlich erleichtert, da alle potentiell

21 Die Zahl am Ende der Referenznachweise bezeichnet die Seitenzahl im Text, d. h. das Zitat bezieht sich auf: Maier (1614, 191-192).

22 Mit wenig über 30 Vorkommen im gesamten Korpus wäre, Ulysses' auch im Close Reading leicht überschaubar. Doch ergibt die automatisierte Analyse lediglich die Kontexte ,mythological ‘ und ,alchemicalopusMeta' und ist damit recht eindeutig. 
relevanten Stellen in der Konkordanz bereits herausgesucht sind. Hinzu kommt der Umstand, dass bei dieser Methode nicht nur der Begriff selbst in Konkordanzansicht vorliegt, sondern auch für alle kontextualisierenden Begriffe automatisch eine solche vorhanden ist. Während dies mithilfe von out-of-the-box-Methoden der quantitativen Textanalyse alles Begriff für Begriff analysiert werden müsste, so kann hier eine ,rekursive` Analyse durchgeführt werden, deren Ergebnisse durch die Wissensressource als Ganze direkt kontextualisiert sind. Für Begriffe können Statistiken erhoben werden, wie häufig sie in welchen Kontexten vorkommen und diese Ergebnisse wiederum in zukünftige Analysen einfließen.

Subjektivität besteht bei dem vorgestellten Vorgehen vor allem in der Auswahl der zu annotierenden Wörter. ${ }^{23}$ Die Kontexte werden zwar durch die Bearbeitenden verschlagwortet und beinhalten damit abermals eine Instanz von Subjektivität, doch erfolgt die Zuteilung der Kontexte daraufhin durch quantitative Auszählung. Damit kann statistisch eruiert werden, welche Begriffe gehäuft mit welchen anderen auftreten. Es entsteht ein Ansatz, der die Methode der Annotation mit quantitativer Textanalyse verbindet. Auch kann über die Konkordanz die Übertragbarkeit des Thesaurus auf andere Texte validiert werden. So kann die Statistik, in welchen Kontexten ein Begriff in Text A vorkommt, mit den Analysewerten aus Text B verglichen werden. Stimmen sie nicht überein, so muss eruiert werden, ob die Begriffsverwendung hier so unterschiedlich ist, dass eine Übertragbarkeit nicht als gegeben angesehen werden kann. Der Thesaurus ist erweiterbar, d. h. er bietet die Möglichkeit, sich zu einem Zeitpunkt in der Zukunft der Abbildung aller möglichen Elemente zumindest anzunähern. Zum aktuellen Zeitpunkt passiert die Auswahl genauer auszuarbeitender Elemente anhand von Häufigkeit: Die häufigsten 50 Konzepte sollen genauer ausgearbeitet werden. Zusätzlich sollen einige für die Forschungsgeschichte besonders relevante Begriffe, wie sie sich in ihrer Wichtigkeit in zeitgenössischen und historischen Alchemie-Lexika und -wörterbüchern niederschlagen, modelliert werden, auch wenn sie weniger häufig sind. Andere in Maiers Indices vorkommende Begriffe werden dennoch annotiert, aber es wird aus pragmatischen Gründen weniger Zeit aufgewendet, sie im Thesaurus tiefenzuerschließen.

Das analytische Auslesen von Kontexten zu Begriffen, die sich aus dem Text selbst ergeben, kann zur Reduktion von Unsicherheit und Ambiguität genutzt werden, womit es zur Disambiguierung beiträgt (Zur Definition von Unsicherheit: Piotrowski 2019). Dies entspricht dem alchemischen Konzept der Wissensdispersi-

23 Vorsicht geboten ist bei der unhinterfragten Verwendung des Subjektivitätsbegriffs. In diesem Fall ist damit durch ForscherInnen getroffene Auswahl und menschliche, methodisch geleitete Intervention gemeint, die von nicht notwendigerweise begründeten Entscheidungen einzelner Akteure zu differenzieren ist. 
on, in dem ein Buch das andere erklären soll. Der vorgestellte Ansatz soll GeisteswissenschaftlerInnen nicht nur erlauben ,digitally enhanced‘ zu arbeiten, sondern soll ganz im Sinne der Digital Humanities eine ,digitally inspired‘ Methode zur Analyse alchemischer Sprache bieten (vgl. Wettlaufer 2016).

\section{Literatur}

Baudrillard, Jean. Mots de Passe. Paris: Pauvert. 2000.

Beynon, Meurig, Steve Russ und Willard McCarty. Human Computing - Modelling with Meaning. In: Literary and Linguistic Computing 21/2. Oxford: Oxford University Press. 2006. DOI: 10.1093/llc/fql015.

Collins, Harry. Tacit and Explicit Knowledge. Chicago: University of Chicago Press. 2010.

Denning, Peter J. The Science of Computing: What Is Computer Science? In: American Scientist 73/1. Research Triangle Park, NC: Sigma Xi, The Scientific Research Honor Society. 1985, S. 16-19.

Duncan, A. M. Styles of Language and Modes of Chemical Thought. In: Ambix 28/2. Milton Park, Abingdon: Taylor \& Francis. 1981, S. 83-107.

Eco, Umberto. II Discorso Alchemico E II Segreto Differito. In: I Limiti Dell'interpretazione (Prima Edizione 1990). Milan: La nave di Teseo. 2016, S. 97-116.

Forshaw, Peter J. Michael Maier and Mythoalchemy. In: Tara Nummedal und Donna Bilak (Hrsg.), Furnace and Fugue. A Digital Edition of Michael Maier's Atalanta fugiens (1618) with Scholarly Commentary. 2020. URL: https://furnaceandfugue.org/essays/forshaw/ (14.08.2020).

Frietsch, Ute. Alchemie Thesaurus. Herzog August Bibliothek Wolfenbüttel. 2017a. URL: http://alchemie.hab.de/thesaurus (06.01.2020).

Frietsch, Ute. Alchemische Fachgruppen. Herzog August Bibliothek Wolfenbüttel. 2017b. URL: http://alchemie.hab.de/alchemische-fachgruppen (06.01.2020).

Frietsch, Ute. Obscurum Vocabulum: Begriffe Der frühneuzeitlichen Alchemie und der AlchemieThesaurus der Herzog August Bibliothek. In: Petra Feuerstein-Herz und Ute Frietsch (Hrsg.), Alchemie - Genealogie Und Terminologie, Bilder, Techniken Und Artefakte. Forschungen Aus Der Herzog August Bibliothek. Wiesbaden. 2021 (noch nicht erschienen).

Gibson, Abraham, Manfred D. Laubichler und Jane Maienschein. Introduction. Isis 110/3. Chicago: University of Chicago Press. 2019, S. 497-501.

Gius, Evelyn und Janina Jacke. Informatik und Hermeneutik. Zum Mehrwert interdisziplinärer Textanalyse. In: Constanze Baum und Thomas Stäcker (Hrsg.), Grenzen und Möglichkeiten der Digital Humanities (Sonderband der Zeitschrift für Digitale Geisteswissenschaften, 1). Wolfenbüttel. 2015. DOI: 10.17175/sb001_006.

Johnson, Kyle P. et al. CLTK: The Classical Language Toolkit. 2014-2019. DOI: 10.5281/zenodo. 593336.

Lang, Sarah. Ein religionswissenschaftlicher Kommentar zu den Arcana Arcanissima und der Mythoalchemie des alchemo-hermetischen latrochemikers Michael Maier (1568-1622). Graz: Leykam. 2018.

Leibenguth, Erik. Hermetische Philosophie des Frühbarock. Die Cantilenae Intellectuales Michael Maiers. Edition mit Übersetzung, Kommentar und Bio-Bibliographie. Tübingen: Niemayer. 2002. 
Lippmann, Edmund Oskar von. Entstehung und Ausbreitung der Alchemie. Mit einem Anhange: Zur älteren Geschichte der Metalle. Ein Beitrag zur Kulturgeschichte. Band 1. Berlin: Springer. 1919.

Maier, Michael. Arcana Arcanissima. London. 1614.

Maier, Michael. Symbola Aureae Mensae Duodecim Nationum. Frankfurt. 1617.

Maier, Michael. Viatorium. Oppenheim. 1618.

McCarty, Willard. Depth, Markup and Modelling. In: Digital Studies/le Champ Numérique 8. 2003. DOI: $10.16995 /$ dscn.167.

McCarty, Willard. Modeling: A Study in Words and Meanings. In: Susan Schreibmann, Ray Siemens und John Unsworth (Hrsg.), A Companion To Digital Humanities. Oxford: Wiley Blackwell. 2004, S. 254-272.

McCarty, Willard. Beyond the Word: Modelling Literary Context. In: Lisa Charlong, Alan Burke und Brad Nickerson (Hrsg.), Text Technology. 2007. URL: http://www.mccarty.org.uk/essays/ McCarty,\%20Beyond\%20the\%20word.pdf (06.01.2020).

Newman, William R. „Decknamen or Pseudochemical Language“? Eirenaeus Philalethes and Carl Jung. In: Revue d'histoire des Sciences 49. Paris: Armand Colin. 1996, S. 159-188.

Newman, William R. und Lawrence M. Principe. Alchemy Vs. Chemistry: The Etymological Origins of a Historiographic Mistake. In: Early Science and Medicine 3/1. Leiden: Brill. 1998, S. 32-65.

Oldman, Dominic, Martin Doerr und Stefan Gradmann. Zen and the Art of Linked Data: New Strategies for a Semantic Web of Humanist Knowledge. In: Susan Schreibmann, Ray Siemens und John Unsworth (Hrsg.), A New Companion To Digital Humanities. Oxford: Wiley Blackwell. 2016, S. 251-273.

Piotrowski, Michael. Accepting and Modeling Uncertainty. In: Andreas Kuczera, Thorsten Wübbena und Thomas Kollatz (Hrsg.), Die Modellierung des Zweifels - Schlüsselideen und -Konzepte zur Graphbasierten Modellierung von Unsicherheiten (= Zeitschrift für Digitale Geisteswissenschaften/Sonderbände, 4). 2019. DOI: 10.17175/sb004_006a.

Polanyi, Michael. The Tacit Dimension (1966). Chicago, London: University of Chicago Press. 2009.

Priesner, Claus und Karin Figala. Vorwort der Herausgeber. In: Claus Priesner und Karin Figala (Hrsg.), Alchemie. Lexikon einer hermetischen Wissenschaft. München: C.H. Beck. 1998, S. 7-11.

Principe, Lawrence M. Robert Boyle's Alchemical Secrecy: Codes, Ciphers and Concealments. In: Ambix 39/2. 1992, S. 63-75. DOI: 10.1179/amb.1992.39.2.63.

Principe, Lawrence M. The Secrets of Alchemy. Chicago: University of Chicago Press. 2013.

Principe, Lawrence M. und William R. Newman. Some Problems with the Historiography of Alchemy. In: William R. Newman und Anthony Grafton (Hrsg.), Secrets of Nature: Astrology and Alchemy in Early Modern Europe. Cambridge, MA: MiT Press. 2001, S. 385-432.

Ruland, Martin. Lexicon Alchemiae Sive Dictionarivm Alchemisticvm: Cum obscuriorum Verborum, \& Rerum Hermeticarum, tum Theophrast-Paracelsicarum Phrasium, Planam Explicationem continens. Francofurtensium Repub.: Palthenius. 1612. URL: https://reader.digitalesammlungen.de/de/fs1/object/display/bsb10220440_00005.html (06.01.2020).

Ruska, Julius und E. Wiedemann. Alchemistische Decknamen. Beiträge zur Geschichte der Naturwissenschaften LXVii, Sitzungsberichte der Phys.-Med. Sozietät Erlangen 56. 1924, S. 17-36.

Schütt, Hans-Werner. Sprachschichten der Alchemie. In: Berichte zur Wissenschaftsgeschichte 17/2. 1994, S. 89-99. DOI: 10.1002/bewi.19940170204. 
Stock, Wolfgang G. und Mechtild Stock. Wissensrepräsentation. Informationen Auswerten und Bereitstellen. München: Oldenbourg. 2008.

Tilton, Hereward. The Quest for the Phoenix. Spiritual Alchemy and Rosicrucianism in the Work of Count Michael Maier (1569-1622). Berlin, New York: De Gruyter. 2003.

W3C-Consortium. Simple Knowledge Organization System (SKOS). 2012. URL: https://www.w3. org/2004/02/skos/ (06.01.2020).

Wettlaufer, Jörg. Neue Erkenntnisse durch digitalisierte Geschichtswissenschaft(en)? Zur hermeneutischen Reichweite aktueller digitaler Methoden in informationszentrierten Fächern. In: Zeitschrift für digitale Geisteswissenschaften. 2016. DOI: 10.17175/2016_011.

Wilhelm, Thomas, Manuel Burghardt und Christian Wolff. „To See or Not to See“ - An Interactive Tool for the Visualization and Analysis of Shakespeare Plays. In: Susan Schreibmann und Ray Siemens und John Unsworth (Hrsg.), Kultur und Informatik: Visual Worlds \& Interactive Spaces. Glückstadt: Verlag Werner Hülsbusch. 2013, S. 175-185. URN: urn:nbn:de:bvb:355epub-284173.

Yu, Liyang. A Developer's Guide to the Semantic Web. Second Edition. Heidelberg: Springer. 2015. 
